\title{
Grape seed polyphenol extract and cognitive function in healthy young adults: A randomised, placebo-controlled, parallel-groups acute-on-chronic trial
}

Article

Accepted Version

Bell, L., Whyte, A. R., Lamport, D. J. ORCID:

https://orcid.org/0000-0002-4592-0439, Spencer, J. P. E., Butler, L. T. and Williams, C. M. ORCID: https://orcid.org/00000003-4452-671X (2020) Grape seed polyphenol extract and cognitive function in healthy young adults: A randomised, placebo-controlled, parallel-groups acute-on-chronic trial. Nutritional Neuroscience, 25 (1). pp. 54-63. ISSN 1028-415X doi: https://doi.org/10.1080/1028415X.2019.1708033 Available at https://centaur.reading.ac.uk/88613/

It is advisable to refer to the publisher's version if you intend to cite from the work. See Guidance on citing.

To link to this article DOI: http://dx.doi.org/10.1080/1028415X.2019.1708033

Publisher: Taylor \& Francis

All outputs in CentAUR are protected by Intellectual Property Rights law, including copyright law. Copyright and IPR is retained by the creators or other copyright holders. Terms and conditions for use of this material are defined in 
the End User Agreement.

www.reading.ac.uk/centaur

\section{CentAUR}

Central Archive at the University of Reading

Reading's research outputs online 


\section{Grape Seed Polyphenol Extract and Cognitive Function in Healthy Young Adults: a randomised, placebo-controlled, parallel-groups acute-on-chronic trial}

Lynne Bell ${ }^{\mathrm{a} \gamma}$, Adrian R Whyte ${ }^{\mathrm{a} \dagger}$, Daniel J Lamport ${ }^{\mathrm{a}}$, Jeremy PE Spencer ${ }^{\mathrm{b}}$, Laurie T Butler ${ }^{\mathrm{c}}$, Claire M Williams ${ }^{\mathrm{a}^{*}}$

${ }^{a}$ School of Psychology and Clinical Language Sciences, University of Reading, Reading, UK. ${ }^{b}$ School of Chemistry, Food and Pharmacy, University of Reading, Reading, UK. ${ }^{c}$ Faculty of Science and Engineering, Anglia Ruskin University, Cambridge, UK.

†joint first author

*corresponding author email: claire.williams@reading.ac.uk

Clinicaltrials.gov identifier: NCT03579095

This research was supported by funding from Polyphenolics, Madera, CA, USA, a division of Constellation Brands, Inc.

\section{Notes on contributors}

Lynne Bell is a postdoctoral researcher working in the Nutritional Psychology laboratory at the School of Psychology \& Clinical Language Sciences, University of Reading, UK.

Adrian Whyte is a postdoctoral researcher working in the Nutritional Psychology laboratory at the School of Psychology \& Clinical Language Sciences, University of Reading, UK.

Daniel J. Lamport is a Lecturer in cognition and nutrition at the School of Psychology \& Clinical Language Sciences, University of Reading, UK. Daniel's research interests include the effect of diet, nutritional interventions, and metabolic disorders on neuropsychological function, and prevention of neurodegenerative disease. 
Jeremy P.E. Spencer is Professor of Molecular Nutrition at the Department of Food and Nutritional Sciences, University of Reading, UK. Jeremy's research is focused on investigating the molecular mechanisms that underlie the accumulating body of epidemiological and medical anthropological evidence on a positive correlation between the consumption of diets rich in fruits and vegetables and a decreased risk of neurodegenerative disorders.

Laurie T. Butler is Pro Vice-Chancellor and Dean of Science of Engineering at Anglia Ruskin University, Cambridge, UK. Laurie is also a visiting Professor at Tohoku University, Japan and at University of Reading, UK. His research interests include nutrition and cognition, ageing, memory and choice, and behaviour change.

Claire M. Williams is Chair of Neuroscience in the School of Psychology \& Clinical Language Sciences at the University of Reading, UK. Her research group, the Nutritional Psychology laboratory, investigates the health benefits of plant-derived chemicals. The focus of her laboratory is the interplay between dietary intake and measures of psychological well-being such as cognitive performance, food preference, mood, and quality of life using animal studies, randomised controlled trials, and neuroimaging across different populations. 


\section{Grape Seed Polyphenol Extract and Cognitive Function in Healthy Young Adults: a randomised, placebo-controlled, parallel-groups acute-on-chronic trial}

Grapes are polyphenol-rich, and grape juice intake has shown cognitive benefits in middle-aged females and older adults with mild cognitive impairment. Extracts obtained from grape seeds have similarly been associated with cognitive benefits in older adults. The aim of this research was to investigate whether a highly purified grape seed-derived polyphenol extract was associated with cognitive benefits in healthy young adults following a single acute dose, and chronically following repeated daily dosage over 12 weeks. Following an acute-on-chronic, parallel groups, randomised, double-blind, placebo-controlled design, sixty adults aged 18-30 consumed either a $400 \mathrm{mg}$ grape seed polyphenol extract (GSPE, $n=30)$ or a placebo $(n=30)$. Cognitive function was assessed acutely at baseline and 2, 4 and 6 h post consumption, and chronically at 6 and 12 twelve weeks with a computerised battery of multiple cognitive tests. Mood was assessed with the Positive and Negative Affect Schedule. Linear marginal model analysis with baseline included as a covariate did not reveal a consistent pattern of cognitive benefits following the GSPE relative to the placebo either acutely or chronically when considering all outcome measures. GSPE was associated with some improvements in reaction time (acutely) and psychomotor skill (chronically), however the placebo was also associated with some benefits to reaction time and memory. Therefore, a $400 \mathrm{mg}$ GSPE did not consistently improve cognitive function in healthy young adults. These findings suggest that younger, healthy populations are perhaps less sensitive to polyphenol extract doses $<400 \mathrm{mg}$ relative to older, or cognitively compromised populations. 


\section{Introduction}

Epidemiological studies suggest a positive association between intake of polyphenolrich fruits, such as grapes, and benefits to cognitive function (1). This is supported by systematic reviews of polyphenol human intervention studies which indicate that single acute doses (2) and chronic daily consumption over several weeks (3) of polyphenol rich-foods has neuropsychological benefits. Grapes (Vitis Vinifera) contain a range of polyphenols (4) which can be categorised into flavonoids (flavan-3-ols, flavonols, anthocyanins and flavones), and non-flavonoids (phenolic acids and stilbenes). Numerous health benefits have been associated with grape polyphenols relating to antiinflammatory properties, cardiovascular outcomes, improved insulin sensitivity, reduced risk of type 2 diabetes, and neuroprotective mechanisms (5). For example, in rodent models supplementation with an extract containing grape and blueberry was associated with increased brain derived neurotrophic factor (BDNF; a protein which contributes to neuroplasticity; (6)), and increased motor and memory functions have been observed in healthy aged rats following intake of grape-derived products $(7,8)$. Further support for a neuroprotective mechanism of action (see (9) for review) comes from positive correlations between increased hippocampal neurogenesis in rodents and presence of grape-derived polyphenols and their metabolites in the bloodstream (6). In humans, BDNF concentrations have been shown to increase following one month of daily grape juice consumption (10) and cognitive benefits have been shown in a number of randomised controlled trials. For example, regular daily grape juice over three months was shown to improve performance on both a neuropsychological test battery and measures of driving performance in stressed middle-aged working mothers (11), and improved memory function in older adults with mild cognitive impairment $(12,13)$. A single acute dose of grape juice has also been associated with improved attention in 
healthy young adults (14). Despite the apparent cognitive benefits for grape juice, there are limited studies using grape seed extracts; the seeds can contain up to $70 \%$ of the extractable polyphenols found in whole grapes (15), thus it can be hypothesised that these extracts contain sufficient polyphenols to induce effects seen with the whole juice. A grape-seed extract (from Vitis Vinifera) has been shown to improve some aspects of cognitive function in older adults aged 55-75 years. This included subtle improvements (relative to baseline) in an assessment designed to detect mild cognitive impairment and dementia (the Mini Mental State Examination) and aspects of language, attention and delayed memory on a test battery following daily intake of the $250 \mathrm{mg}$ extract over twelve weeks. Aspects of mood were also found to improve, including reduced depression and anxiety scores. Whilst there is, therefore, tentative evidence that grape seed extracts may benefit the brain in older adults, further investigation is required to explore whether these benefits extend to younger populations, and also build on evidence from grape juice, and other aforementioned polyphenol studies, to explore whether acute as well as chronic effects exist. Therefore, the aim of the present study was to investigate whether a highly purified grape seed-derived polyphenolic extract from Vitis Vinifera was associated with cognitive benefits following a single acute dose, and chronically following repeated daily dosage over 12 weeks (with mid-point testing at 6 weeks) in healthy young adults.

\section{Method}

\section{Design}

The study was registered on ClinicalTrials.Gov (Identifier NCT03526406). An acuteon-chronic, parallel groups, randomised, double-blind, placebo-controlled design was implemented. Participants were randomly allocated to the grape seed polyphenol extract 
(GSPE) or placebo condition using an established randomisation procedure. To maintain double-blinding the extracts were labelled with codes by the manufacturer, which was revealed to the research team following completion of the analysis. For the acute design, participants were tested at baseline (prior to treatment), and at two, four and six hours post treatment consumption. For the chronic design participants were tested at baseline (Visit 1; prior to treatment), six weeks (Visit 2) and twelve weeks (Visit 3) following daily treatment. Intervention compliance was monitored through the use of a self-report form where participants recorded days when they had forgotten to take their allotted intervention capsule. Remaining capsules were counted when participants returned for testing at Visit 2 and Visit 3, and numbers were cross-checked with the self-report form. For both the acute and chronic design, outcome measures were cognitive function and mood. A favourable opinion for conduct was attained from the University of Reading School of Psychology and Clinical Language Sciences Research Ethics Committee and this study follows the principles of the Declaration of Helsinki.

\section{Intervention}

The GSPE and the placebo were identical in appearance and delivered in capsule form (made of bovine gelatine), each matched for weight and containing $400 \mathrm{mg}$ powder. The exact calorie conternt of each was unknown. The GSPE was a $400 \mathrm{mg}$ mix of purified grape seed-derived polyphenolic extracts from Vitis Vinifera (MegaNatural AZ; Polyphenolics Inc., Madera, CA, USA) containing catechin, epicatechin, proanthocyanidins, and derivatives of catechin and epicatechin (epicatechin gallate) based on in-house analysis by Polyphenolics Inc. The placebo contained $400 \mathrm{mg}$ maltodextrin. Both the GSPE and placebo powders contained trace amounts of modified cellulose, magnesium stearate, and silica. 


\section{Participants}

Sixty healthy participants (GSPE $n=30$ and placebo $n=30$ ) aged 18-30 were recruited via opportunistic sampling. Sample size was determined by power analysis using GPower 3.1., assuming a medium effect size (2), 30 participants per intervention group were needed to detect a significant difference between groups with an alpha of 0.05 and $80 \%$ statistical power. Exclusion criteria were any diagnosed disease or illness, including psychiatric or neurological conditions, consumption of any medication or nutritional supplements, participation in any clinical trial within previous 6 months, alcohol consumption >14 units/week, smoking, vegetarian or vegan, pregnancy, >4 hours/week vigorous exercise. All participants were required to give informed consent before taking part.

\section{Outcome measures}

Cognitive function was assessed with a computerised battery of tests administered in a fixed order lasting approximately 45 minutes including the Auditory Verbal Learning Test (AVLT), Serial Subtraction 3s and 7s, the Modified Attention Network Test (ANT), Simple and Complex Finger Tapping and the Switching Task.

The AVLT required participants to listen to a recording of 15 words (list A) following which there was a 2 minutes period to orally recall as many words as possible. This was repeated a further 4 times, followed by presentation and recall of a new 'interference' list (List B), immediately followed by a final recall of List A. Following a 25 minute period during which the remaining cognitive tests were presented, delayed recall of List A took place, followed by a recognition task whereby participants distinguished List A words from List B words and an additional 20 previously unseen foils. Different word lists, matched for concreteness and familiarity, were administered in a counterbalanced 
order at each test session. Dependent variables were the total recall at each repetition, delayed recall, learning (List A recall 5 - List A recall 1) proactive interference (List A recall 1 - List B recall 1), retroactive interference (List A recall 5 - List A recall 6), and number of words correctly recognised.

Serial Subtraction $3 \mathrm{~s}$ and $7 \mathrm{~s}$ provided a measure of working memory, whereby a random number between 800 and 999 was presented on screen and participants subtracted backwards (in 3s or 7s), answering using a number pad as quickly as possible over 2 minutes. Dependent variables were number of correct responses and errors.

The ANT is an executive function task whereby participants viewed blocks of arrows presented on screen in rapid succession and indicated with a key press (left or right arrow) the direction of the arrow closest to a central fixation point. The target arrow was either flanked by arrows pointing in the same (congruent) or opposite (incongruent) direction, or was not flanked at all. On selected trials, spatial cues were introduced immediately prior to the appearance of the arrows. Task load was further manipulated by increasing or decreasing the number of flanking arrows across multiple trials. Participants completed 36 practice trials, followed by 160 trials in the main task. Dependent variables were reaction time and accuracy.

Simple Finger Tapping involved pressing the "c" key as many times as possible with the index finger of the dominant hand for a duration of 30 s. Complex Finger Tapping followed the same procedure except the required response was "c","b", "v", "n" using the first, third, second and fourth fingers, respectively (or the reverse for left handed participants). The dependent variables for this psychomotor function task were the total number of correct responses for simple tapping, and the total number of correct sequences for complex finger tapping. 
The Switching Task assessed executive function. Participants viewed eight equally spaced radii of a circle displayed in such a way that there were four equally spaced segments above and below a bold line. A stimulus digit selected from between $1-9$ (excluding 5) appeared in each segment in turn in a clockwise direction. Each digit was displayed for a duration of $3000 \mathrm{~ms}$, or until the participant responded. Inter-stimulus interval was $500 \mathrm{~ms}$. Dependent on whether the stimulus was in the segments above or below the bold line, participants performed different tasks. If the number was above the bold line, participants discerned whether the stimulus was odd or even by pressing the relevant response key, whereas if the number was below the bold line, participants discerned whether the number was higher or lower than 5, again by pressing the relevant key. The task switched every 4 trials. For the first 8 rotations of the task, participants received feedback on all errors or failures to respond within 3 seconds. This data was excluded from the final analysis. Participants then completed 48 rotations of the task (containing 96 switch trials and 588 non-switch trials) during which no feedback was provided. Dependent variables were accuracy and reaction time.

Subjective mood was assessed with the PANAS-Now questionnaire, whereby participants rated 20 mood adjectives on a scale of 1-5, giving two dependent variables Positive and Negative Affect. Mental fatigue was also assessed with a nine point Likert scale. These subjective mood variables were recorded at the beginning and end of the test battery. A change score was calculated by subtracting the end ratings from those recorded at the beginning. Average daily fruit and vegetable consumption was assessed using data from the EPIC-Norfolk Food Frequency Questionnaire. BMI (kg/m2) was calculated from height and weight measurements taken once at the beginning of the practice visit. 


\section{Procedure}

Participants attended the University of Reading School of Psychology for four separate visits; Practice Visit, Visit 1 (week 0), Visit 2 (week 6) and Visit 3 (week 12). For 48 hours prior to each test visit, participants followed a low polyphenol diet requiring restriction of foods including fruits, vegetables, juices, chocolate, tea $\&$ coffee (caffeinated beverages were only restricted for 24 hours). Compliance was recorded with a food diary. Participants were also required to be fasted for 12 hours. Upon arrival (8am-9am) participants consumed a low polyphenol breakfast of two croissants, consumed within 15 minutes. At the Practice Visit a practice version of the cognitive battery (data not recorded) and mood measures were completed. Participants completed the EPIC-Norfolk FFQ to record their habitual diet, and height and weight measures were taken. At Visit 1,2 and 3 the mood measures and cognitive battery were administered immediately after breakfast. At Visit 1 , the first treatment was consumed following completion of the baseline cognitive battery (at Visits 2 and 3 participants consumed their treatment immediately after completion of the mood/cognitive measures). At Visit 1 only, a standardised lunch (cheese sandwich and crisps) was provided at the end of the $2 \mathrm{~h}$ cognitive battery, and two further mood/cognitive assessments were administered 4 and 6 hours post treatment consumption. During all breaks participants resumed their normal daily activities but were asked to avoid all food and beverages other than water, and to avoid vigorous exercise. After testing was completed, participants were provided a supply of capsules, and returned 6 weeks later for Visit 2. At the end of Visit 3 the EPIC- Norfolk food frequency questionnaire was completed (only taking into account their diet during the 12 week study period) and participants received a $£ 100$ honorarium. 


\section{Statistical analysis}

For the chronic analysis, a linear marginal model (LMM) using an unstructured covariance matrix to model Visit 2 (week 6) and Visit 3 (week 12) with baseline Visit 1 (week 0) as a repeated covariate was applied. Visit and Intervention Group were included as fixed factors in the model. For analysis of the acute data, an unstructured covariance matrix to model two, four and six hours, with baseline as a repeated covariate was applied. Time and Intervention Group were included as fixed factors in the model. Post hoc pairwise comparisons were used to investigate any significant effects of Intervention Group. A Bonferroni correction was applied to all multiple comparisons. For all RT data, only correct responses were included. Z score analysis was used to identify outliers (z score>3.29 were removed prior analysis; (16). All outcome measures met the required assumptions of normality. Raw data from this project will be openly available from the University of Reading Research Data Archive at http://researchdata.reading.ac.uk/.

\section{Results}

Sixty participants completed the study. The CONSORT diagram is shown in Figure 1. Table 1 outlines sample characteristics, for which there were no significant differences between treatment groups.

[Figure 1 here]

[Table 1 here]

\section{Acute analysis}

The majority of outcomes showed no significant effects of treatment (see Table 2).

There was evidence that GSPE was associated with faster responses for the Switching Task across all time points, as indicated by a main effect of treatment 
$(\mathrm{F}[1,459]=10.13, \mathrm{p}<.01)$. This was further supported by the significant treatment*time interaction $(\mathrm{F}[2,453]=3.31, \mathrm{p}<.05)$ with pairwise comparisons showing significantly faster RT for GSPE relative to placebo at $2 \mathrm{hrs}$ and $4 \mathrm{hrs}$ (both $\mathrm{p}<.001$ ). GSPE consumption was also associated with benefits for reaction time on the ANT; pairwise comparisons showed faster responses at $6 \mathrm{hrs}$ relative to $4 \mathrm{hrs}(\mathrm{p}<.001)$ following a treatment*time interaction $(\mathrm{F}[2,431)=4.45, \mathrm{p}<.05)$. Faster ANT RT was also seen between $2 \mathrm{hrs}$ and $4 \mathrm{hrs}$ following placebo $(\mathrm{p}<.001)$, and better performance was observed following the placebo relative to the GSPE for AVLT Total Recall $(\mathrm{F}[1,56]=5.56, \mathrm{p}<.05)$, Retroactive Interference $(\mathrm{F}[1,54]=4.17, \mathrm{p}<.05)$, and Switching Task accuracy $(\mathrm{F}[1,435]=12.19, \mathrm{p}<.01]$ as indicated by main effects of treatment (see Table 2).

[Table 2 here]

\section{Chronic analysis}

Similarly to the acute analysis, the majority of outcomes showed no significant effects of treatment (see Table 3). There was evidence of a GSPE benefit for Simple Finger Tapping (treatment*visit interaction $\mathrm{F}[1,60]=8.36, \mathrm{p}<.01$ ) whereby performance decreased between weeks 6 and 12 for the placebo $(\mathrm{p}<.05)$, with performance maintained for GSPE. A treatment*visit interaction was also observed for AVLT Total Recall $(\mathrm{F}[1,53]=6.28, \mathrm{p}<.05)$ with performance better for the placebo group relative to the GSPE group at week 6 ( $p<.05)$, however, GSPE performance increased between week 6 and $12(\mathrm{p}<.05)$, to a level commensurate with the placebo group. Unexpectedly, there was evidence for a slowing in reaction time between weeks 6 and 12 for the GSPE on the Switching Task $(\mathrm{p}<.01)$ and the ANT $(\mathrm{p}<.01)$ as indicated by significant treatment*visit interactions $(\mathrm{F}[1,448)=7.62, \mathrm{p}<.01)$ and $(\mathrm{F}[1,444]=25.66, \mathrm{p}<.001)$ respectively. Table 3 shows that a number of outcomes showed significant main effects 
of visit. These showed that subjective mood improved between week 6 and 12 as indicated by higher ratings of positive affect and lower ratings of fatigue (both $\mathrm{p}<.05$ ). Interestingly, cognitive performance declined on some outcomes between weeks 6 and 12 as shown by lower accuracy coupled with slower reactions times for the ANT and the Switching Task (all, $\mathrm{p}<.05)$.

\section{[Table 3 here]}

\section{Discussion}

In summary, there was limited evidence for cognitive benefits following acute or chronic consumption of a 400mg Vitis Vinifera grape seed polyphenol extract in healthy adults aged 18-30. Acutely, there were improvements in reaction time 2-4 hours post consumption of the extract for two measures of executive function (switching task and the ANT), and following chronic dosage of the extract faster finger tapping was observed. However, a number of (acute and chronic) positive effects were observed following the placebo, and when considering the range of cognitive outcomes assessed there were no consistent benefits of the extract. The reaction time and finger tapping effects that were associated with the extract can be broadly described as psychomotor function. Interestingly, improvements in finger tapping have also been shown in healthy young adults following acute flavonoid-rich orange juice intervention, without benefits for a number of other cognitive measures (17). Similarly, a grape juice intervention in healthy young adults did not show effects on individual cognitive tests, but benefits were observed for a composite measure of attention reaction time (14). Psychomotor function is a lower order cognitive process which contributes to any cognitive measure involving reaction time, and it is plausible that psychomotor function improvements will precede benefits on other more complex, higher order cognitive functions, such as executive function inhibition tasks (e.g. the ANT and switching task here). In support, a 
review of acute flavonoid interventions (2) showed that benefits are most consistently seen for attention, working memory, and psychomotor processing speed. Here, the polyphenol (and flavonoid) dose may have been too small to induce observable benefits on the majority of the complex measures of executive function and episodic memory, but it may have been sufficient to produce the small, albeit inconsistent effects for psychomotor function. However, discussions and interpretations relating to the present dose are limited as the exact concentration of polyphenols (or sub-classes) in the grape seed extract were not quantified. What is known is that the dose was $<400 \mathrm{mg}$ given the total size of the extract.

Limited and null cognitive effects of polyphenol interventions in healthy young adults have also been reported elsewhere, including resveratrol supplements (18), green tea epigallocatechin gallate extracts (19) and a single serving of grape juice (20). Healthy young adults, who are at peak cognitive capacity with regards to the lifespan maybe less likely to benefit from polyphenol interventions relative to older adults in cognitive decline, or children who are experiencing cognitive development. In support, polyphenol-rich grape juice has been shown to improve cognitive function in middleaged, stressed females (11) and older adults with mild cognitive impairment $(12,13)$. This indicates that grape juice and its constituents (e.g. polyphenols) can benefit the brain, particularly in adults with cognitive decline, potentially via neuroprotective mechanisms such as improved synaptic plasticity, anti-inflammatory actions, and reduced neurodegeneration (9). In support, cerebral physiological benefits have been detected following six months grape juice supplementation in healthy older adults; cerebral metabolism was seen to decline following the placebo, but these reductions were attenuated following the grape juice (21). Furthermore, thirty days grape juice supplementation was found to increase levels of BDNF in healthy older adults (10). 
These mechanistic actions will arguably have lower efficacy in healthy (young) populations who do not show increased neuronal oxidative stress or neurodegeneration. However, even in older adults, these physiological changes may not always manifest observable behavioural changes $(10,21)$.

Population differences may, therefore, contribute to inconsistencies between the current findings and a twelve week trial of grape seed extract supplementation in older adults aged 56-75 years (22), where improvements in general cognitive function were found, as assessed with the Mini-Mental State Examination and specific aspects of a neuropsychological test battery including attention and memory. However, their nonparametric analysis model did not consider change from baseline for the extract relative to change from baseline for the placebo, and baseline performance for extract and placebo were not directly compared. The results therefore warrant further validation. This highlights the importance of applying appropriate and rigorous analysis models, such as linear mixed and marginal models, which can account for baseline performance as a covariate, as applied here. There are numerous recommendations for conducting controlled trials of nutritional interventions for health outcomes such as cognitive function (e.g. (23)), which should be considered for developing a strong evidence base and addressing calls for increasing reproducibility and replication (24).

A further point of note is that polyphenol supplements may be subject to degradation over time. Some of the unexpected findings in the current study could be explained by such a degradation of the GSPE capsules. Participants were not given specific storage instructions for the capsules, and remaining capsules were not tested for polyphenol content at the end of the study. Future work should consider such a measure or include the testing of metabolites in plasma or urine throughout the study to continually monitor bioactivity. An additional limitation of the current study is that the young adult 
population tested was biased towards females. However, analysis of the exclusively female sample did not change the reported outcomes.

Despite the limited evidence here, future research is required to understand the efficacy of grape seed polyphenol extracts for cognitive benefits in humans, given that there is supportive evidence from rodent studies (e.g. $(7,8))$ and human studies with grape juice (e.g. (11-14)). Concomitant assessment of behavioural outcomes with consideration of the bioavailability of grape polyphenols when consumed as an extract, or indeed as whole juice, would advance mechanistic understanding. For example, a review of the brain distribution of grape-derived phenolics and metabolites following supplementation in rodents indicated that circulation in the blood stream and accumulation in brain tissue is low (25). Dose response studies would give an indication of the concentrations required to observe behavioural effects, as has been documented with peripheral vascular outcomes following flavonoid-rich blueberries (26). The important contribution of the gut microbiota for determining individual differences in absorption, distribution, metabolism, excretion, bioavailability, and subsequently cognitive outcomes has also been highlighted (25). For example, in diabetic rodents, the extent of grape polyphenol metabolites found in the plasma and brain tissue following supplementation was associated with specific metabolising enzymes in the gut (27). There are also likely to be age related differences in the permeability of the blood brain barrier (28) and its susceptibility to phenolic metabolites (29), which could contribute to the apparently greater cognitive sensitivity of older adults relative to younger adults following grape juice and extract supplementation. Future work should examine this, and investigate whether the specific phenolics present in grape extract (e.g. flavan-3-ols, flavonols, anthocyanins, flavones, phenolic acids, and stilbenes) have different contributions. It is also currently unclear how behavioural effects may differ between 
supplementation from grape juice and grape seed extracts. Currently, this cannot be answered as this is one of only two grape seed extract cognitive function studies in humans (also (22)), however, it can be hypothesised than any differences will be associated with variations in the digestion, metabolism, and subsequent bioavailability of whole fruits, juices and extracts. To conclude, this is the first study to specifically examine cognitive effects of grape seed extracts in healthy young adults. There were no clear, consistent cognitive benefits relative to placebo consumption either acutely over six hours or following six and twelve weeks daily consumption. Further research should consider the role of dose, population and individual differences in bioavailability of grape seed extract. Given current interest in the facilitation of healthy aging, investigation of the optimum doses for the mitigation of cognitive decline in older adults appears a priority.

\section{Disclosure of interest}

The authors report no conflict of interest. The study was funded by Constellation Brands, Inc. However, no representative from the company had any input into the study design, implementation, analysis or interpretation of the data.

\section{References}

1. Kesse-Guyot E, Andreeva VA, Jeandel C, Ferry M, Hercberg S, Galan P. A healthy dietary pattern at midlife is associated with subsequent cognitive performance. The Journal of nutrition. 2012;142(5):909-15.

2. Bell L, Lamport DJ, Butler LT, Williams CM. A Review of the Cognitive Effects Observed in Humans Following Acute Supplementation with Flavonoids, and Their Associated Mechanisms of Action. Nutrients. 2015;7(12):10290-306. 
3. Lamport DJ, Dye L, Wightman JD, Lawton CL. The effects of flavonoid and other polyphenol consumption on cognitive performance: a systematic research review of human experimental and epidemiological studies. Nutrition and Aging. 2012;1(1):525.

4. Garrido J, Borges F. Wine and grape polyphenols-A chemical perspective. Food research international. 2013;54(2):1844-58.

5. Nassiri-Asl M, Hosseinzadeh H. Review of the pharmacological effects of Vitis vinifera (Grape) and its bioactive compounds. Phytotherapy Research: An International Journal Devoted to Pharmacological and Toxicological Evaluation of Natural Product Derivatives. 2009;23(9):1197-204.

6. Dal-Pan A, Dudonne S, Bourassa P, Bourdoulous M, Tremblay C, Desjardins Y, et al. Cognitive-enhancing effects of a polyphenols-rich extract from fruits without changes in neuropathology in an animal model of Alzheimer's disease. Journal of Alzheimer's Disease. 2017;55(1):115-35.

7. Sarkaki A, Farbood Y, Badavi M. The effect of grape seed extract (GSE) on spatial memory in aged male rats. Pakistan Journal of Medical Sciences. 2007;23(4):561.

8. Shukitt-Hale B, Carey A, Simon L, Mark DA, Joseph JA. Effects of Concord grape juice on cognitive and motor deficits in aging. Nutrition. 2006;22(3):295-302. 9. Rendeiro C, Rhodes JS, Spencer JP. The mechanisms of action of flavonoids in the brain: direct versus indirect effects. Neurochemistry International. 2015;89:126-39. 10. Trevizol L, Bassôa L, Fraga I, Munhoz I, Pochmann D, Dani C, et al. Grape juice consumption and/or exercise training-induced neuroplasticity and memory improvement in healthy elderly women. 
11. Lamport DJ, Lawton CL, Merat N, Jamson H, Myrissa K, Hofman D, et al. Concord grape juice, cognitive function, and driving performance: a 12-wk, placebocontrolled, randomized crossover trial in mothers of preteen children. The American journal of clinical nutrition. 2016;103(3):775-83.

12. Krikorian R, Boespflug EL, Fleck DE, Stein AL, Wightman JD, Shidler MD, et al. Concord grape juice supplementation and neurocognitive function in human aging. Journal of agricultural and food chemistry. 2012;60(23):5736-42.

13. Krikorian R, Nash TA, Shidler MD, Shukitt-Hale B, Joseph JA. Concord grape juice supplementation improves memory function in older adults with mild cognitive impairment. Br J Nutr. 2010;103(5):730-4.

14. Haskell-Ramsay C, Stuart R, Okello E, Watson A. Cognitive and mood improvements following acute supplementation with purple grape juice in healthy young adults. European journal of nutrition. 2017;56(8):2621-31.

15. Shi J, Yu J, Pohorly JE, Kakuda Y. Polyphenolics in grape seeds-biochemistry and functionality. Journal of medicinal food. 2003;6(4):291-9.

16. Tabachnick BG, Fidell LS. Using multivariate statistics, 6th edn Boston. Ma: Pearson. 2013.

17. Alharbi MH, Lamport DJ, Dodd GF, Saunders C, Harkness L, Butler LT, et al. Flavonoid-rich orange juice is associated with acute improvements in cognitive function in healthy middle-aged males. European Journal of Nutrition. 2016;55(6):2021-9. 18. Kennedy DO, Wightman EL, Reay JL, Lietz G, Okello EJ, Wilde A, et al. Effects of resveratrol on cerebral blood flow variables and cognitive performance in humans: a double-blind, placebo-controlled, crossover investigation. The American journal of clinical nutrition. 2010;91(6):1590-7. 
19. Wightman EL, Haskell CF, Forster JS, Veasey RC, Kennedy DO.

Epigallocatechin gallate, cerebral blood flow parameters, cognitive performance and mood in healthy humans: a double-blind, placebo-controlled, crossover investigation. Human Psychopharmacology: Clinical and Experimental. 2012;27(2):177-86.

20. J. Hendrickson S, D. Mattes R. No acute effects of grape juice on appetite, implicit memory and mood. Food \& nutrition research. 2008;52(1):1891.

21. Lee J, Torosyan N, Silverman DH. Examining the impact of grape consumption on brain metabolism and cognitive function in patients with mild decline in cognition: a double-blinded placebo controlled pilot study. Experimental gerontology. 2017;87:1218.

22. Calapai G, Bonina F, Bonina A, Rizza L, Mannucci C, Arcoraci V, et al. A Randomized, double-blinded, clinical trial on effects of a Vitis vinifera extract on cognitive function in healthy older adults. Frontiers in pharmacology. 2017;8:776. 23. Welch RW, Antoine J-M, Berta J-L, Bub A, de Vries J, Guarner F, et al. Guidelines for the design, conduct and reporting of human intervention studies to evaluate the health benefits of foods. British Journal of Nutrition. 2011;106(S2):S3-S15. 24. Sorkin BC, Kuszak AJ, Williamson JS, Hopp DC, Betz JM. The challenge of reproducibility and accuracy in nutrition research: resources and pitfalls. Advances in Nutrition. 2016;7(2):383-9.

25. Zhao D, Simon JE, Wu Q. A critical review on grape polyphenols for neuroprotection: Strategies to enhance bioefficacy. Critical reviews in food science and nutrition. 2019:1-29.

26. Rodriguez-Mateos A, Rendeiro C, Bergillos-Meca T, Tabatabaee S, George TW, Heiss C, et al. Intake and time dependence of blueberry flavonoid-induced improvements in vascular function: a randomized, controlled, double-blind, crossover 
intervention study with mechanistic insights into biological activity. The American journal of clinical nutrition. 2013;98(5):1179-91.

27. Chen TY, Ferruzzi MG, Wu QL, Simon JE, Talcott ST, Wang J, et al. Influence of diabetes on plasma pharmacokinetics and brain bioavailability of grape polyphenols and their phase II metabolites in the Zucker diabetic fatty rat. Molecular nutrition \& food research. 2017;61(10):1700111.

28. Abbott NJ, Patabendige AA, Dolman DE, Yusof SR, Begley DJ. Structure and function of the blood-brain barrier. Neurobiology of disease. 2010;37(1):13-25.

29. Margalef M, Pons Z, Iglesias-Carres L, Quiñones M, Bravo FI, Arola-Arnal A, et al. Rat health status affects bioavailability, target tissue levels, and bioactivity of grape seed flavanols. Molecular nutrition \& food research. 2017;61(2):1600342. 


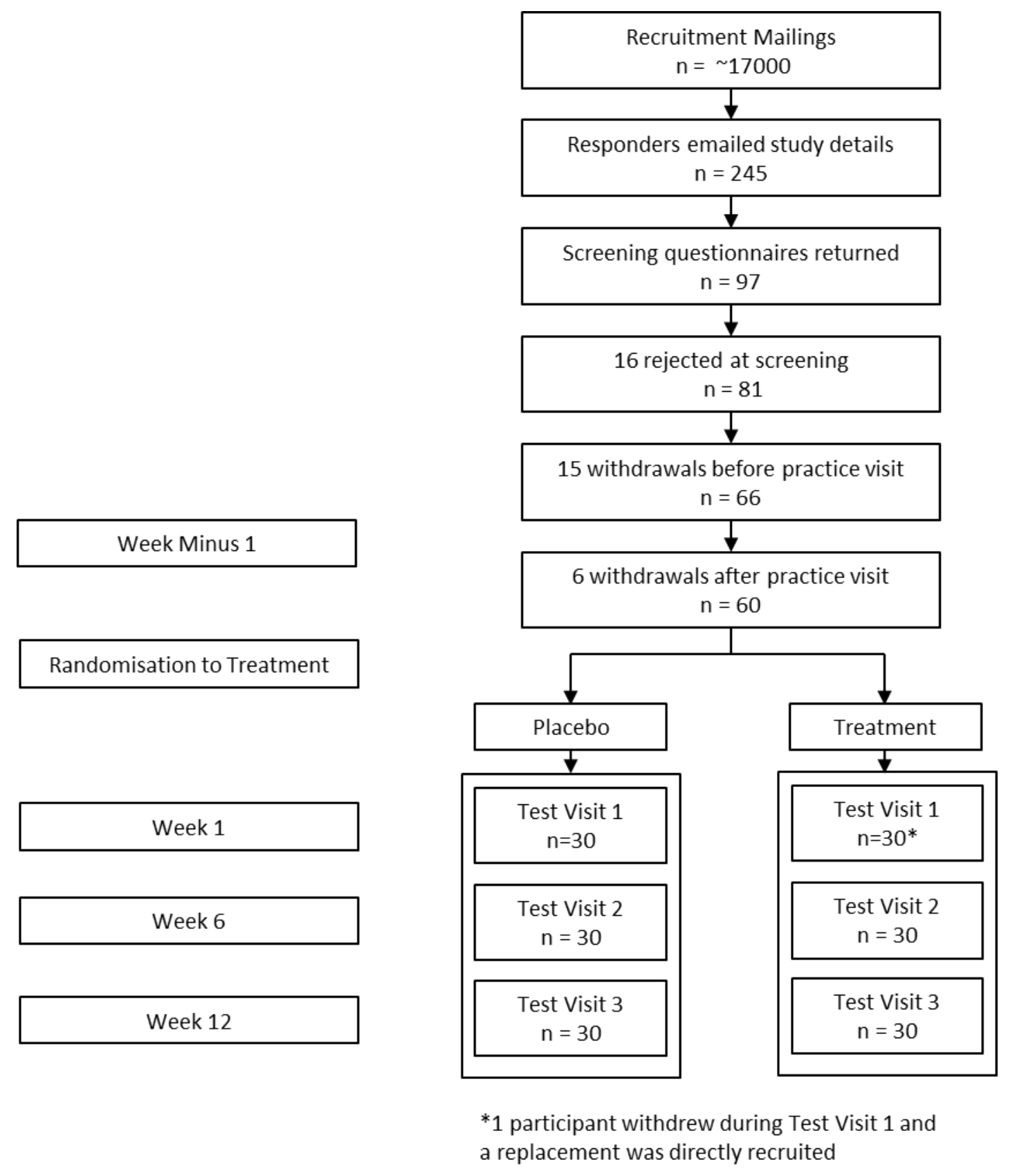

Figure 1 CONSORT Diagram 
Table 1. Sample characteristics at enrolment.

\begin{tabular}{lllllllll}
\hline & \multicolumn{3}{c}{ Placebo } & \multicolumn{5}{c}{ Grape Seed } \\
Polyphenol Extract & \\
Characteristic & Mean & SD & Range & Mean & SD & Range & p- \\
value
\end{tabular}


Table 2. Acute data and LMM summary.

\begin{tabular}{|c|c|c|c|c|c|c|c|c|c|c|c|c|c|c|c|}
\hline \multirow[b]{3}{*}{ Task } & \multicolumn{6}{|c|}{ Placebo } & \multicolumn{6}{|c|}{ Grape Seed Polyphenol Extract } & & & \\
\hline & \multicolumn{2}{|c|}{$2 \mathrm{~h}$} & \multicolumn{2}{|c|}{$4 \mathrm{~h}$} & \multicolumn{2}{|c|}{$6 \mathrm{~h}$} & \multicolumn{2}{|c|}{$2 \mathrm{~h}$} & \multicolumn{2}{|c|}{$4 \mathrm{~h}$} & \multicolumn{2}{|c|}{$6 \mathrm{~h}$} & \multicolumn{3}{|c|}{ LMM Fixed Effects p-values } \\
\hline & Mean & SE & Mean & $\mathrm{SE}$ & Mean & $\mathrm{SE}$ & Mean & SE & Mean & $\mathrm{SE}$ & Mean & SE & Time & Treatment & $\begin{array}{l}\text { Treatment } \mathrm{x} \\
\text { Time }\end{array}$ \\
\hline \multicolumn{16}{|l|}{ PANAS (out of 50): } \\
\hline Positive affect 1 & 22.26 & 0.77 & 20.09 & 0.79 & 20.09 & 0.99 & 22.49 & 0.79 & 19.63 & 0.80 & 19.66 & 1.00 & $<0.001$ & $\mathrm{~ns}$ & ns \\
\hline Positive affect 2 & 18.35 & 0.63 & 17.18 & 0.72 & 18.68 & 0.86 & 18.62 & 0.63 & 16.68 & 0.72 & 18.22 & 0.86 & 0.004 & $\mathrm{~ns}$ & $\mathrm{~ns}$ \\
\hline Positive affect change & -4.17 & 0.70 & -3.17 & 0.81 & -1.67 & 0.94 & -4.55 & 0.71 & -3.48 & 0.83 & -1.76 & 0.96 & 0.040 & $\mathrm{~ns}$ & ns \\
\hline Negative affect 1 & 11.34 & 0.25 & 11.03 & 0.24 & 11.41 & 0.23 & 11.22 & 0.25 & 11.36 & 0.24 & 11.40 & 0.24 & ns & $\mathrm{ns}$ & ns \\
\hline Negative affect 2 & 12.08 & 0.38 & 11.56 & 0.33 & 11.68 & 0.36 & 11.87 & 0.38 & 12.42 & 0.33 & 12.94 & 0.36 & ns & $(0.080)$ & $\mathrm{ns}$ \\
\hline Negative affect change & 0.69 & 0.34 & 0.67 & 0.30 & 0.35 & 0.29 & 0.42 & 0.34 & 0.93 & 0.30 & 1.15 & 0.30 & ns & $\mathrm{ns}$ & ns \\
\hline \multicolumn{16}{|l|}{ Mental Fatigue (out of 9): } \\
\hline Fatigue 1 & 3.72 & 0.23 & 4.22 & 0.27 & 4.72 & 0.32 & 3.75 & 0.23 & 4.28 & 0.27 & 5.25 & 0.32 & $<0.001$ & $\mathrm{~ns}$ & ns \\
\hline Fatigue 2 & 5.71 & 0.26 & 6.41 & 0.28 & 5.97 & 0.31 & 5.93 & 0.26 & 6.36 & 0.28 & 6.79 & 0.31 & 0.016 & $\mathrm{~ns}$ & ns \\
\hline Fatigue change & 1.94 & 0.32 & 2.14 & 0.30 & 1.21 & 0.27 & 2.23 & 0.32 & 2.13 & 0.30 & 1.59 & 0.27 & 0.029 & $\mathrm{~ns}$ & $\mathrm{~ns}$ \\
\hline \multicolumn{16}{|c|}{ Serial $3 s \& 7 s$ (in 2 minutes): } \\
\hline Serial 3s score & 44.50 & 1.44 & 47.34 & 2.10 & 48.80 & 1.91 & 45.20 & 1.47 & 46.76 & 2.13 & 46.27 & 1.95 & 0.024 & $\mathrm{~ns}$ & $\mathrm{~ns}$ \\
\hline Serial 3s errors & 2.32 & 0.33 & 1.99 & 0.28 & 1.52 & 0.39 & 1.91 & 0.33 & 1.94 & 0.27 & 2.61 & 0.39 & ns & $\mathrm{ns}$ & 0.017 \\
\hline Serial 7s score & 21.94 & 0.89 & 22.94 & 1.04 & 25.04 & 1.07 & 23.68 & 0.91 & 24.25 & 1.04 & 24.49 & 1.07 & 0.020 & $\mathrm{~ns}$ & $\mathrm{~ns}$ \\
\hline Serial 7s errors & 2.62 & 0.40 & 2.79 & 0.35 & 2.13 & 0.33 & 3.05 & 0.40 & 3.23 & 0.35 & 2.43 & 0.33 & 0.017 & $\mathrm{~ns}$ & ns \\
\hline \multicolumn{16}{|c|}{ Finger tapping (in 30 secs): } \\
\hline Simple & 121.94 & 1.38 & 122.31 & 1.44 & 123.21 & 1.56 & 123.55 & 1.40 & 121.37 & 1.47 & 122.24 & 1.59 & ns & $\mathrm{ns}$ & ns \\
\hline Complex & 21.09 & 0.50 & 21.99 & 0.60 & 21.96 & 0.71 & 21.24 & 0.49 & 21.11 & 0.59 & 21.91 & 0.69 & $\mathrm{~ns}$ & $\mathrm{~ns}$ & ns \\
\hline \multicolumn{16}{|l|}{ AVLT recall (out of 15 ): } \\
\hline AVLT Immediate recall & 6.21 & 0.30 & 6.42 & 0.30 & 6.42 & 0.32 & 5.77 & 0.31 & 6.11 & 0.31 & 6.25 & 0.33 & $\mathrm{~ns}$ & $\mathrm{~ns}$ & $\mathrm{~ns}$ \\
\hline AVLT Final recall & 13.29 & 0.27 & 13.46 & 0.22 & 13.25 & 0.29 & 13.06 & 0.28 & 13.22 & 0.23 & 13.30 & 0.31 & ns & $\mathrm{ns}$ & ns \\
\hline
\end{tabular}




\begin{tabular}{|c|c|c|c|c|c|c|c|c|c|c|c|c|c|c|c|}
\hline \multirow[b]{3}{*}{ Task } & \multicolumn{6}{|c|}{ Placebo } & \multicolumn{6}{|c|}{ Grape Seed Polyphenol Extract } & & & \\
\hline & \multicolumn{2}{|c|}{$2 \mathrm{~h}$} & \multicolumn{2}{|c|}{$4 \mathrm{~h}$} & \multicolumn{2}{|c|}{$6 \mathrm{~h}$} & \multicolumn{2}{|c|}{$2 \mathrm{~h}$} & \multicolumn{2}{|c|}{$4 \mathrm{~h}$} & \multicolumn{2}{|c|}{$6 \mathrm{~h}$} & \multicolumn{3}{|c|}{ LMM Fixed Effects p-values } \\
\hline & Mean & SE & Mean & SE & Mean & SE & Mean & SE & Mean & SE & Mean & $\mathrm{SE}$ & Time & Treatment & $\begin{array}{l}\text { Treatment } \mathrm{x} \\
\text { Time }\end{array}$ \\
\hline AVLT Total recall (out of 75) & 54.62 & 1.17 & 54.48 & 1.21 & 54.17 & 1.47 & 50.59 & 1.21 & 50.07 & 1.26 & 51.74 & 1.53 & $\mathrm{~ns}$ & 0.022 & ns \\
\hline AVLT Learning & 7.02 & 0.34 & 6.98 & 0.35 & 6.78 & 0.41 & 7.31 & 0.35 & 6.94 & 0.36 & 6.83 & 0.42 & $\mathrm{~ns}$ & $\mathrm{~ns}$ & $\mathrm{~ns}$ \\
\hline AVLT Proactive interference & 1.06 & 0.34 & 0.58 & 0.38 & 0.61 & 0.39 & 1.19 & 0.36 & 0.97 & 0.39 & 1.22 & 0.41 & $\mathrm{~ns}$ & ns & ns \\
\hline AVLT Retroactive interference & 1.32 & 0.29 & 1.11 & 0.29 & 1.26 & 0.36 & 1.80 & 0.30 & 2.09 & 0.30 & 1.80 & 0.37 & ns & 0.046 & ns \\
\hline AVLT Delayed recall & 10.16 & 0.52 & 10.33 & 0.53 & 9.81 & 0.60 & 9.42 & 0.54 & 9.31 & 0.55 & 9.09 & 0.63 & $\mathrm{~ns}$ & $\mathrm{~ns}$ & $\mathrm{~ns}$ \\
\hline \multicolumn{16}{|l|}{ AVLT recognition: } \\
\hline Yes responses (out of 15) & 12.95 & 0.27 & 12.45 & 0.31 & 12.75 & 0.34 & 12.49 & 0.27 & 12.16 & 0.31 & 12.28 & 0.35 & ns & ns & ns \\
\hline No reponses (out of 35) & 33.95 & 0.23 & 33.93 & 0.23 & 34.30 & 0.17 & 34.17 & 0.24 & 34.07 & 0.23 & 34.27 & 0.17 & $\mathrm{~ns}$ & $\mathrm{~ns}$ & ns \\
\hline \multicolumn{16}{|l|}{ ANT: } \\
\hline Accuracy $(\%)$ & 0.97 & $<0.01$ & 0.96 & $<0.01$ & 0.97 & $<0.01$ & 0.97 & $<0.01$ & 0.96 & $<0.01$ & 0.97 & $<0.01$ & 0.036 & $\mathrm{~ns}$ & $\mathrm{~ns}$ \\
\hline Reaction time (ms) & 449.82 & 5.31 & 437.87 & 5.30 & 435.31 & 5.24 & 451.17 & 5.22 & 448.29 & 5.22 & 439.03 & 5.18 & $<0.001$ & ns & 0.012 \\
\hline \multicolumn{16}{|l|}{ Switching task: } \\
\hline Accuracy $(\%)$ & 0.95 & $<0.01$ & 0.95 & $<0.01$ & 0.95 & $<0.01$ & 0.94 & $<0.01$ & 0.93 & $<0.01$ & 0.94 & $<0.01$ & 0.003 & 0.001 & $(0.051)$ \\
\hline Reaction time (ms) & 764.79 & 6.78 & 760.32 & 8.42 & 724.39 & 7.98 & 732.25 & 6.78 & 721.47 & 8.42 & 714.01 & 7.88 & $<0.001$ & 0.002 & 0.037 \\
\hline
\end{tabular}

Reported values are estimated marginal means with baseline as covariate; ns indicates non-significant effect p $>0.10$, brackets around p-value indicate non-significant trend $(0.05<\mathrm{p}<0.10)$ 
Table 3. Chronic data and LMM summary.

\begin{tabular}{|c|c|c|c|c|c|c|c|c|c|c|c|}
\hline \multirow[b]{3}{*}{ Task } & \multicolumn{4}{|c|}{ Placebo } & \multicolumn{4}{|c|}{ Grape Seed Polyphenol Extract } & & & \\
\hline & \multicolumn{2}{|c|}{$6 w k$} & \multicolumn{2}{|c|}{$12 \mathrm{wk}$} & \multicolumn{2}{|c|}{$6 \mathrm{wk}$} & \multicolumn{2}{|c|}{$12 \mathrm{wk}$} & \multicolumn{3}{|c|}{ LMM Fixed Effects p-values } \\
\hline & Mean & SE & Mean & $\mathrm{SE}$ & Mean & $\mathrm{SE}$ & Mean & SE & Visit & Treatment & Treatment $\mathrm{x}$ Visit \\
\hline \multicolumn{12}{|l|}{ PANAS (out of 50): } \\
\hline Positive affect 1 & 22.88 & 0.99 & 24.68 & 1.07 & 23.49 & 0.99 & 25.43 & 1.07 & 0.016 & ns & ns \\
\hline Positive affect 2 & 19.64 & 1.11 & 21.00 & 1.09 & 18.89 & 1.13 & 20.18 & 1.12 & $(0.074)$ & ns & ns \\
\hline Positive affect change & -2.74 & 0.88 & -3.09 & 0.89 & -4.33 & 0.88 & -4.60 & 0.89 & ns & ns & ns \\
\hline Negative affect 1 & 12.17 & 0.51 & 12.05 & 0.58 & 13.09 & 0.50 & 12.92 & 0.57 & ns & ns & ns \\
\hline Negative affect 2 & 12.00 & 0.54 & 12.31 & 0.60 & 13.59 & 0.53 & 14.15 & 0.59 & ns & 0.015 & ns \\
\hline Negative affect change & -0.19 & 0.51 & -0.02 & 0.42 & 0.49 & 0.51 & 1.22 & 0.42 & ns & $(0.066)$ & ns \\
\hline \multicolumn{12}{|l|}{ Mental Fatigue (out of 9): } \\
\hline Fatigue 1 & 4.35 & 0.32 & 3.39 & 0.34 & 4.35 & 0.32 & 3.81 & 0.34 & 0.023 & ns & ns \\
\hline Fatigue 2 & 5.77 & 0.35 & 5.07 & 0.34 & 5.59 & 0.35 & 5.43 & 0.34 & $(0.084)$ & ns & ns \\
\hline Fatigue change & 1.43 & 0.31 & 1.69 & 0.28 & 1.24 & 0.31 & 1.61 & 0.28 & ns & ns & ns \\
\hline \multicolumn{12}{|l|}{ Serial $3 s \& 7 s$ (in 2 minutes): } \\
\hline Serial 3s score & 47.94 & 1.58 & 49.47 & 2.08 & 44.31 & 1.58 & 44.79 & 2.09 & ns & $(0.060)$ & ns \\
\hline Serial 3s errors & 2.06 & 0.38 & 2.66 & 0.40 & 2.28 & 0.38 & 2.25 & 0.40 & ns & ns & ns \\
\hline Serial 7s score & 24.20 & 1.07 & 23.29 & 0.94 & 24.62 & 1.07 & 24.40 & 0.94 & ns & ns & ns \\
\hline Serial $7 \mathrm{~s}$ errors & 2.40 & 0.46 & 2.33 & 0.41 & 2.71 & 0.47 & 2.83 & 0.41 & ns & ns & ns \\
\hline \multicolumn{12}{|l|}{ Finger tapping (in 30 secs): } \\
\hline Simple & 124.36 & 1.66 & 121.43 & 1.73 & 123.24 & 1.66 & 125.71 & 1.73 & ns & ns & 0.005 \\
\hline Complex & 20.48 & 0.73 & 21.14 & 0.67 & 20.57 & 0.72 & 21.03 & 0.65 & ns & ns & ns \\
\hline \multicolumn{12}{|l|}{ AVLT recall (out of 15$)$ : } \\
\hline AVLT Immediate recall & 7.55 & 0.31 & 7.41 & 0.39 & 6.58 & 0.32 & 7.20 & 0.41 & ns & ns & ns \\
\hline AVLT Final recall & 13.94 & 0.22 & 13.73 & 0.25 & 13.56 & 0.23 & 13.74 & 0.26 & $\mathrm{~ns}$ & $\mathrm{~ns}$ & $\mathrm{~ns}$ \\
\hline AVLT Total recall (out of 75) & 59.00 & 1.04 & 57.66 & 1.22 & 54.75 & 1.08 & 57.43 & 1.30 & ns & ns & 0.015 \\
\hline
\end{tabular}




\begin{tabular}{|c|c|c|c|c|c|c|c|c|c|c|c|}
\hline \multirow[b]{3}{*}{ Task } & \multicolumn{4}{|c|}{ Placebo } & \multicolumn{4}{|c|}{ Grape Seed Polyphenol Extract } & & & \\
\hline & \multicolumn{2}{|c|}{$6 w k$} & \multicolumn{2}{|c|}{$12 \mathrm{wk}$} & \multicolumn{2}{|c|}{$6 \mathrm{wk}$} & \multicolumn{2}{|c|}{$12 \mathrm{wk}$} & \multicolumn{3}{|c|}{ LMM Fixed Effects p-values } \\
\hline & Mean & $\mathrm{SE}$ & Mean & $\mathrm{SE}$ & Mean & $\mathrm{SE}$ & Mean & $\mathrm{SE}$ & Visit & Treatment & Treatment $\mathrm{x}$ Visit \\
\hline AVLT Learning & 6.38 & 0.35 & 6.31 & 0.42 & 6.84 & 0.37 & 6.53 & 0.44 & ns & $\mathrm{ns}$ & ns \\
\hline AVLT Proactive interference & -0.17 & 0.41 & 0.04 & 0.36 & 0.12 & 0.42 & 0.40 & 0.39 & ns & ns & ns \\
\hline AVLT Retroactive interference & 0.85 & 0.25 & 0.65 & 0.25 & 0.99 & 0.27 & 1.24 & 0.26 & ns & ns & ns \\
\hline AVLT Delayed recall & 12.78 & 0.36 & 12.64 & 0.32 & 12.01 & 0.37 & 12.29 & 0.34 & ns & ns & ns \\
\hline \multicolumn{12}{|l|}{ AVLT recognition: } \\
\hline Yes responses (out of 15 ) & 13.84 & 0.25 & 13.78 & 0.26 & 13.41 & 0.26 & 13.54 & 0.26 & ns & $\mathrm{ns}$ & ns \\
\hline No responses (out of 35) & 34.58 & 0.16 & 34.41 & 0.18 & 34.46 & 0.16 & 34.51 & 0.17 & ns & ns & ns \\
\hline \multicolumn{12}{|l|}{$A N T$} \\
\hline Accuracy $(\%)$ & 0.98 & $<0.01$ & 0.97 & $<0.01$ & 0.98 & $<0.01$ & 0.97 & $<0.01$ & 0.039 & ns & ns \\
\hline Reaction time (ms) & 443.04 & 4.35 & 441.76 & 4.45 & 449.25 & 4.35 & 457.53 & 4.45 & 0.044 & $(0.072)$ & 0.006 \\
\hline \multicolumn{12}{|l|}{ Switching task: } \\
\hline Accuracy $(\%)$ & 0.96 & 0.01 & 0.95 & 0.01 & 0.95 & 0.01 & 0.94 & 0.01 & $<0.001$ & ns & ns \\
\hline Reaction time (ms) & 746.91 & 20.26 & 732.75 & 20.31 & 748.73 & 19.97 & 792.00 & 20.08 & 0.011 & ns & $<0.001$ \\
\hline
\end{tabular}

Reported values are estimated marginal means with baseline as covariate; ns indicates non-significant effect $\mathrm{p}>0.10$, brackets around $\mathrm{p}$-value indicate nonsignificant trend $(0.05<\mathrm{p}<0.10)$ 